\title{
Plantas medicinais utilizadas pela comunidade do bairro dos Tenentes - município de Extrema, MG, Brasil
}

\author{
COSTA, V.P.; MAYWORM, M.A.S.* \\ Universidade de Santo Amaro, Faculdade de Biologia, Laboratório de Fitoquímica, Rua Professor Enéas de Siqueira \\ Neto, 340, Jardim Imbuias, CEP: 04829-300, São Paulo-Brasil * masmayworm@ig.com.br
}

\begin{abstract}
RESUMO: Este trabalho teve como objetivo verificar junto à comunidade do bairro dos Tenentes (Extrema, MG) como é feito o tratamento de doenças a partir do uso de plantas medicinais, bem como identificar e relacionar as plantas utilizadas com a literatura científica. Para tanto, foram realizadas entrevistas e coletas de plantas utilizadas na medicina popular local as quais, após processo de herborização e identificação, foram comparadas com informações disponíveis em literatura especializada. Foram identificadas 71 espécies, pertencentes a 33 famílias botânicas, sendo Asteraceae e Lamiaceae as mais expressivas. As folhas e ramos (88\%) foram as partes mais utilizadas e a forma de preparo mais freqüente foi a infusão (67\%). As plantas foram indicadas principalmente para o tratamento de males associados ao aparelho digestório (23 espécies), respiratório (16), excretor (10), nervoso (6), circulatório (5), endócrino (4), reprodutor feminino (2), e ainda como cicatrizante (7), antiinflamatório (4) e para dores no corpo (5).
\end{abstract}

Palavras-chave: etnobotânica, conhecimento popular, Minas Gerais

\begin{abstract}
Medicinal plants used by the community of Tenentes District - Extrema Municipality, Minas Gerais State, Brazil. This study aimed to verify with the community of Tenentes District (Extrema Municipality, Minas Gerais State, Brazil) how diseases are treated by using medicinal plants, as well as to identify and relate the used plants to the scientific literature. Thus, interviews were done and plants used in the local folk medicine were collected; after the herborization process and identification, the obtained data were compared with information available in the literature. Seventy-one species were identified; they belonged to 33 botanical families, of which Asteraceae and Lamiaceae were most expressive. Leaves and branches (88\%) were the most used part and the most frequent form of preparation was infusion (67\%). The plants were indicated especially for the treatment of illness associated with digestive (23 species), respiratory (16), excretory (10), nervous (6), circulatory (5), endocrine (4) and woman reproductive systems (2), as healing (7), anti-inflammatory (4), and for general body aches (5).
\end{abstract}

Key words: ethnobotany, folk knowledge, Minas Gerais

\section{INTRODUÇÃO}

As plantas têm sido utilizadas pelo homem há milhares de anos e continuam tendo o seu valor não apenas nas comunidades tradicionais como também são objetos de estudos interdisciplinares na busca de novos fármacos (Macedo et al., 2002).

Segundo Phillips \& Gentry (1993), o uso popular de plantas medicinais é uma técnica baseada no acúmulo de informações repassadas oralmente por sucessivas gerações.

A Organização Mundial da Saúde define planta medicinal como sendo "qualquer vegetal que possui, em um ou mais órgãos, substâncias que podem ser utilizadas com fins terapêuticos ou que sejam precursores de fármacos semi-sintéticos" (WHO,1998).

Os estudos envolvendo o conhecimento e utilizações populares das plantas para os mais diversos fins, entre eles os medicinais, são desenvolvidos pela etnobotânica. Segundo Amorozo (1996), a etnobotânica engloba a maneira como um grupo social classifica as plantas e as utiliza.

O Brasil é um dos maiores centros de biodiversidade vegetal do planeta, com diversos ambientes e floras especificas e abriga centenas de grupos étnicos, que introduziram na cultura popular a

Recebido para publicação em 11/07/2009

Aceito para publicação em 24/01/2011

Rev. Bras. PI. Med., Botucatu, v.13, n.3, p.282-292, 2011. 
utilização de muitas espécies para os mais diversos fins, entre eles o uso medicinal. Além da assimilação dos conhecimentos indígenas, as contribuições trazidas pelos escravos e imigrantes representaram papel importante para o surgimento de uma medicina popular rica. Entre as plantas medicinais que tiveram origem na cultura dos diversos grupos indígenas estão a ipeca, o jaborandi, a carqueja, o guaraná, o taiuiá e a erva-debugre. Muitas outras foram trazidas pelos europeus, como a hortelã, a camomila, a malva, o funcho e pelos africanos, como a erva-guiné e o melão-de-São-Caetano (Simões, 1998).

Segundo Maciel et al. (2002) as observações populares sobre o uso e a eficácia de plantas medicinais contribuem de forma relevante para a divulgação das potencialidades terapêuticas das plantas, e também desperta o interesse de pesquisadores de áreas como a botânica, farmacologia e fitoquímica, enriquecendo 0 conhecimento e intensificando a utilização de muitas plantas. Dessa forma, levantamentos etnobotânicos podem subsidiar estudos etnofarmacológicos na busca por fitoterápicos para o tratamento de úlceras pépticas, inflamações e outras enfermidades (Albuquerque \& Hanazaki, 2006).

Nos últimos anos, o número de trabalhos publicados tem crescido (Oliveira et al., 2009) mostrando a etnobotânica de grupos indígenas e quilombolas (Hanazaki et al., 2000; Rodrigues \& Carlini, 2003; Franco \& Barros, 2006), de comunidades caiçaras (FonsecaKruel \& Peixoto, 2004), bem como de comunidades rurais e de centros urbanos (Rodrigues \& Carvalho, 2001; Pereira et al., 2004; Arnous et al., 2005; Pinto et al., 2006; Cunha-Lima et al., 2008; Jesus et al., 2009; Santos et al., 2009).

Estudos feitos em comunidades urbanas como os quintais de bairros populares nas periferias de grandes centros, ou em núcleos urbanos isolados têm sua importância, pois nestas comunidades ocorre o chamado cultivo "ex situ" de muitas espécies da flora local e de outros lugares, que muitas vezes não ocorrem mais nas áreas naturais devido as mais diversas ações humanas.

Desta forma, este trabalho visou realizar junto à comunidade do bairro dos Tenentes, no município de Extrema, Minas Gerais, um levantamento sobre o uso de plantas medicinais, e comparar essas informações com aquelas obtidas junto à literatura científica.

\section{MATERIALE MÉTODO}

A cidade de Extrema situa-se ao sul do estado de Minas Gerais, em altitude de 973 msm, na divisa dos estados de São Paulo e Minas Gerais, junto à margem da Rodovia BR 381 - Fernão Dias, a 107 km da capital paulista e possui uma área de $242,46 \mathrm{~km}^{2}$. A diversidade de formações vegetais dentro do município acarreta uma expressiva riqueza de espécies, estando a região situada em área de Mata Atlântica. Avegetação, porém, atualmente encontra-se bastante fragmentada devido à intensidade de ocupação sofrida ao longo de pelo menos dois séculos, sendo a fisionomia caracterizada por mosaicos de áreas mais densas e áreas mais abertas. As atividades agropecuárias transformaram grande parte da paisagem do município em vegetações homogêneas constituídas por pastagens, áreas pequenas de lavouras e alguns fragmentos de reflorestamento com eucaliptos (Ribeiro, 2004). De acordo com dados coletados na Prefeitura Municipal de Extrema, o bairro dos Tenentes possui 220 famílias residentes, localizado aproximadamente a $6 \mathrm{~km}$ do centro da cidade. A escolha da área de estudo foi baseada em prévias observações, através das quais foi possível identificar o uso e cultivo de plantas medicinais em muitas propriedades, e a crescente degradação de parte da área de estudo. Foram realizadas oito visitas ao bairro dos Tenentes, em Extrema, Minas Gerais, ao longo de quatro meses, período em que foram coletadas as informações de 50 entrevistados (em 50 residências) em diferentes pontos da área de estudo. Os entrevistados foram selecionados ao acaso, ou às vezes, a partir da recomendação de outros entrevistados. Os entrevistados, 27 mulheres e 23 homens, com idade entre 35 e 70 anos, apresentam baixo nível de escolaridade, desenvolvem atividades domésticas, agricultura de subsistência, pequenos serviços braçais ou são aposentados.

As coletas de dados e das amostras das plantas utilizadas foram feitas no quintal da própria residência de cada entrevistado ou em locais próximos, quando indicados.

Foram realizadas entrevistas semi-estruturadas e estruturadas (Albuquerque \& Lucena, 2004) através da aplicação de um questionário com os dados pessoais dos entrevistados e dados sobre as espécies como: nomes comuns, partes utilizadas, modo de preparo e finalidade de uso. As indicações propostas pela comunidade foram agrupadas de acordo com a classificação do CID-10 - Classificação estatística internacional de doenças e problemas relacionados à saúde (WHO, 2007). O material botânico coletado foi prensado, seco, herborizado e incorporado ao Herbário da Universidade de Santo Amaro (Herbário Unisa), de acordo com Fidalgo \& Bononi (1989). As identificações dos espécimes foram feitas através de comparações com materiais de herbário e consultas a especialista (Sumiko Honda) do Herbário Municipal de São Paulo, e a classificação das famílias foi baseada segundo APG III (Stevens, 2010).

\section{RESULTADO E DISCUSSÃO}

A Tabela 1 apresenta o levantamento das plantas utilizadas pela comunidade do bairro dos Tenentes. Foram coletados 239 espécimes,

Rev. Bras. Pl. Med., Botucatu, v.13, n.3, p.282-292, 2011. 
pertencentes a 62 gêneros, e 71 espécies. A hortelã (Mentha spp.) e a erva-cidreira [Cymbopogon citratus (DC.) Stapf] foram as plantas com o maior número de citações (22 e 20 citações, respectivamente), e Asteraceae (16 espécies) e Lamiaceae (9 espécies) foram as famílias com o maior número de espécies utilizadas, semelhante ao observado em outras comunidades (Pereira et al., 2004; Vendruscolo \& Mentz, 2006).

A maior utilização de espécies de Asteraceae pode ser creditada a grande diversidade de compostos secundários. Um levantamento feito no final da década de 90 apontou mais de 10.000 compostos isolados de Asteraceae (Emerenciano et al., 1998), Nos representantes de Lamiaceae, a presença de óleos voláteis, compostos com ações carminativa, antiespasmódica, secretolítica, antiinflamatória, entre outras (Simões \& Spitzer, 2000), torna essas plantas muito úteis no tratamento de várias enfermidades.

As plantas foram indicadas principalmente para o tratamento de males associados ao aparelho digestório (23 espécies), respiratório (16), excretor (10), nervoso (6), circulatório (5), endócrino (4), reprodutor feminino (2), e ainda como cicatrizante (7), antiinflamatório (4) e para dores no corpo (5).

Entre as plantas citadas para combater infecções do aparelho excretor, Costus spiralis (Jacq.) Roscoe apresenta ações antimicrobiana (Pérez et al., 2008) e urolítica (Viel et al., 1999), Phyllanthus tenellus Roxb e Persea americana Mill. têm atividade antimicrobiana (Oliveira et al., 2007a; Castillo-Juárez et al., 2009), corroborando as indicações dos entrevistados. Não foram encontrados trabalhos sobre a atividade antimicrobiana de Mikania cordifolia (L. f.) Willd., porém segundo Yatsuda et al. (2005), extratos de Mikania laevigata Sch. Bip. ex Baker e $M$. glomerata Spreng. tem atividade antimicrobiana, o que também poderia ocorrer com $M$. cordifolia, apontada pelos entrevistados. Entre as plantas indicadas para tratamento de cálculos renais, a indicação de Phyllanthus tenellus Roxb pode estar correta, pois segundo Campos \& Schor (1999) e Freitas et al. (2002), extratos aquosos de Phyllanthus neruriL. tem ação urolítica.

Para tratamento de problemas relacionados ao aparelho respiratório, várias espécies foram citadas para combater tosse e dor de garganta, enfermidades geradas muitas vezes por ação de microorganismos. Entre essas, estudos desenvolvidos com um fitoterápico contendo extrato fluido de Mikania glomerata e Eucalyptus globulus Labill. mostraram atividade antitussígena em cobaias (Mello \& Mello, 2005). Outras plantas também indicadas para tosse e dor de garganta, tiveram o potencial antimicrobiano confirmado em testes de laboratório, entre as quais estão Punica granatum L. (Naz et al., 2007), Plantago australis Lam. (Lapenna et al., 2003), Mentha arvensis
L., Mentha X piperita L. (Imai et al., 2001), Ruta graveolens L. (Ivanova et al., 2005), Vernonia polyanthes Less. (Oliveira et al., 2007b), Malva sylvestris L. (Cheng \& Wang, 2006) e Rubus rosifolius Sm. (Mauro et al., 2002), corroborando com as indicações propostas pelos entrevistados. Entre as espécies apontadas para 0 tratamento de bronquite, Eucalytus globolus Labill. e Ocimum canum Sims. apresentam óleos voláteis ricos em cineol, e Mentha arvensis apresenta teores elevados de mentol compostos, com atividade expectorante (Simões \& Spitzer, 2000).

Diabetes foi a única doença citada pelos entrevistados relacionada ao sistema endócrino, para qual foram indicadas apenas Bauhinia forficata Linke Cissus verticillata (L.) Nicolson \& C.E. Jarvis, cujas propriedades foram confirmadas através de vários estudos (Pepato et al., 2002; Barbosa et al., 2002; Menezes et al., 2007; Khalil et al., 2008), corroborando a utilização dessas espécies pela comunidade.

Entre as espécies utilizadas para o tratamento de problemas do aparelho digestório, várias plantas indicadas pelos entrevistados para tratamento de "dor de barriga", "dor de estômago", cólica ou "dor no fígado" já foram avaliadas quanto aos seus potenciais antiespasmódico e/ou analgésico. Entre essas estão Cymbopogon citratus (Lorenzetti et al., 1991), Plectranthus barbatus Andrews (Câmara et al., 2003), Ageratum conyzoides L. (Margot \& Silva et al., 2000), Baccharis trimera L. (Gene et al., 1996), Psidium guajava L. (Lozoya et al., 1994; Tona et al., 2000), Kalanchoe pinnata (Lam.) Pers. (Mans et al., 2004), Achillea millefolium L. (Lemmens-Gruber, 2006), Maytenus aquifolium Mart. (Gonzalez et al., 2001), Sedum dendroideum Moc \& Sessé ex DC. (De Melo et al., 2005), Vernonia condensata Baker (Frutuoso et al., 1994), Petiveria alliacea L. (Lopes-Martins et al., 2002). Estudos com o extrato de Stachytarpheta cayennensis (Rich.) Vahl evidenciaram a redução de secreção gástrica em cobaias (Mesia-Vela et al., 2004), corroborando assim as informações coletadas junto aos entrevistados. Outras espécies foram citadas para o tratamento de disenteria ou diarréia, quadros clínicos causados por microorganismos. Entre as plantas apontadas estão Ficus carica L. que apresenta atividade antimicrobiana (Jeong et al., 2009; Aref et al., 2010) e Psidium guajava cuja ação antidiarreica já foi comprovada (Salgado et al., 2006). Chenopodium ambrosioides L. e Mentha sp foram indicadas para tratamentos de verminoses. A ação anti-helmíntica de C. ambrosioides é associada ao alto teor de ascaridol, presente no óleo essencial das folhas (Sousa et al., 1991). Alguns trabalhos demonstraram que o extrato de Mentha x piperita tem atividade antiparasitária (Naranjo et al., 2006; Vidal et al., 2007), o que também poderia ocorrer com as outras espécies do gênero, corroborando as indicações dessas plantas para o combate de verminoses. 
TABELA 1. Espécies utilizadas como medicinais pela comunidade do bairro dos Tenentes, Extrema, MG.

\begin{tabular}{|c|c|c|c|c|c|}
\hline $\begin{array}{l}\text { Familia } \\
\text { Espécie }\end{array}$ & $\begin{array}{l}\text { Nome } \\
\text { comum }\end{array}$ & $\begin{array}{l}\mathrm{N} \text { 으 de } \\
\text { coleta }\end{array}$ & $\begin{array}{c}\text { Parte } \\
\text { utilizada }\end{array}$ & $\begin{array}{l}\text { Formas } \\
\text { de } \\
\text { preparo }\end{array}$ & Indicações \\
\hline $\begin{array}{l}\text { Alismataceae } \\
\text { Echinodorus macrophyllus (Kunth) } \\
\text { Mitch. }\end{array}$ & $\begin{array}{l}\text { chapéu-de- } \\
\text { couro }\end{array}$ & $\begin{array}{c}\text { Costa } \\
60\end{array}$ & Fo & In & Cálculo renal \\
\hline $\begin{array}{l}\text { Amaranthaceae } \\
\text { Chenopodium ambrosioides L. }\end{array}$ & $\begin{array}{c}\text { mastruz, } \\
\text { erva-de-santa } \\
\text { maria }\end{array}$ & $\begin{array}{l}\text { Costa } \\
245\end{array}$ & Fo & In, Ct, De & Vermífugo \\
\hline $\begin{array}{l}\text { Anacardiaceae } \\
\text { Mangifera indica L. } \\
\text { Schinus terebinthifolia Raddi }\end{array}$ & aroeira & $\begin{array}{l}\text { Costa } \\
41 \\
\text { Costa } \\
147 \\
\end{array}$ & $\mathrm{Ca}, \mathrm{Fo}$ & $\mathrm{De}, \mathrm{Ct}$ & $\begin{array}{l}\text { Úlcera, infecções, bronquite } \\
\text { Antibiótico, cicatrizante }\end{array}$ \\
\hline $\begin{array}{l}\text { Asphodelaceae } \\
\text { Aloe arborescens Mill. } \\
\text { A. vera (L.) Burm. f. }\end{array}$ & $\begin{array}{l}\text { babosa-de- } \\
\text { árvore } \\
\text { babosa }\end{array}$ & $\begin{array}{l}\text { Costa } \\
155 \\
\text { Costa } \\
62\end{array}$ & Fo & $\begin{array}{l}\mathrm{Xa} \\
\mathrm{Na}\end{array}$ & $\begin{array}{l}\text { Limpeza do corpo } \\
\text { Tosse, gastrite, aftas } \\
\text { Couro cabeludo, } \\
\text { queimaduras }\end{array}$ \\
\hline $\begin{array}{l}\text { Asteraceae } \\
\text { Acanthospermum australe (Loefl.) } \\
\text { Kuntze }\end{array}$ & carrapichinho & $\begin{array}{c}\text { Costa } \\
43\end{array}$ & Fo & In & Febre \\
\hline Achillea millefolium L. & novalgina & $\begin{array}{c}\text { Costa } \\
231\end{array}$ & Fo & In & $\begin{array}{l}\text { Dor de cabeça, dor de } \\
\text { barniga }\end{array}$ \\
\hline Ageratum conyzoides L. & $\begin{array}{l}\text { mentrato, } \\
\text { mentraste, } \\
\text { erva-de-são- } \\
\text { joão }\end{array}$ & $\begin{array}{r}\text { Costa } \\
244\end{array}$ & FI, Fo & $\mathrm{BA}, \mathrm{De}, \mathrm{In}$ & $\begin{array}{l}\text { Brotoeja, gases, dor de } \\
\text { barniga }\end{array}$ \\
\hline Artemisia absinthium L. & losna & $\begin{array}{l}\text { Costa } \\
53\end{array}$ & Fo & $\mathrm{De}, \ln$ & $\begin{array}{l}\text { Ânsia de vômito, dor de } \\
\text { estômago }\end{array}$ \\
\hline A. annua L. & artimeio & $\begin{array}{c}\text { Costa } \\
139\end{array}$ & Fo & In & Dor no corpo, febre \\
\hline A. canphorata Vill. & canfora & $\begin{array}{l}\text { Costa } \\
176\end{array}$ & Fo & $\mathrm{Al}$ & Dor muscular, hematoma \\
\hline Baccharis trimera (Less.) DC. & carqueja & $\begin{array}{c}\text { Costa } \\
14\end{array}$ & $\mathrm{Ca}, \mathrm{Ra}$ & In, $\mathrm{Na}$ & $\begin{array}{l}\text { Fígado, dor de estômago, } \\
\text { rim, gastrite, par a emagrecer }\end{array}$ \\
\hline Bidens pilosa L. & $\begin{array}{l}\text { picão, picão } \\
\text { preto }\end{array}$ & $\begin{array}{c}\text { Costa } \\
71\end{array}$ & Fo, Fl & $\mathrm{De}, \ln$ & Fígado, hepatite \\
\hline $\begin{array}{l}\text { Chamomilla recutita (L.) } \\
\text { Rauschert }\end{array}$ & camomila & $\begin{array}{l}\text { Costa } \\
206\end{array}$ & $\mathrm{FI}$ & De, In & Calmante \\
\hline Galinsoga parviflora Cav. & picão-branco & $\begin{array}{c}\text { Costa } \\
23\end{array}$ & Fo & $\ln$ & Fígado \\
\hline Mikania cordifolia (L. f.) Willd. & cipó-cabeludo & $\begin{array}{l}\text { Costa } \\
15\end{array}$ & Fo & $\ln$ & Infecção urinária \\
\hline M. glomerata Spreng. & guaco & $\begin{array}{c}\text { Costa } \\
93\end{array}$ & Fo & $\ln$ & Tosse \\
\hline Mikania sp(1) & guaco & $\begin{array}{c}\text { Costa } \\
10\end{array}$ & Fo & In, Xa & Gripe tosse \\
\hline Mikania sp(2) & guaco & $\begin{array}{c}\text { Costa } \\
47\end{array}$ & Fo & $\mathrm{Xa}$ & $\begin{array}{l}\text { Tosse, infecção urinária, } \\
\text { gripe }\end{array}$ \\
\hline
\end{tabular}


TABELA1. Espécies utilizadas como medicinais pela comunidade do bairro dos Tenentes, Extrema, MG.

\begin{tabular}{|c|c|c|c|c|c|}
\hline $\begin{array}{l}\text { Família } \\
\text { Espécie }\end{array}$ & $\begin{array}{l}\text { Nome } \\
\text { comum }\end{array}$ & $\begin{array}{l}\text { No- de } \\
\text { coleta }\end{array}$ & $\begin{array}{c}\text { Parte } \\
\text { utilizada }\end{array}$ & $\begin{array}{c}\text { Form as } \\
\text { de } \\
\text { preparo }\end{array}$ & Indicações \\
\hline \multicolumn{6}{|l|}{ Asteraceae } \\
\hline $\begin{array}{l}\text { Porophyllum ruderale (Jacq.) } \\
\text { Cass. }\end{array}$ & arnica & $\begin{array}{c}\text { Costa } \\
11\end{array}$ & Fo & $\mathrm{Al}$ & Antiinflamatório \\
\hline Solva pterosperma (Juss.) Less. & marcelinha & $\begin{array}{c}\text { Costa } \\
107\end{array}$ & Fo & In & Dor de barriga \\
\hline Vernonia condensata Baker & $\begin{array}{l}\text { boldo } \\
\text { paraguaio }\end{array}$ & $\begin{array}{c}\text { Costa } \\
197\end{array}$ & Fo & In, Ma & Mal estar, dor de estômago \\
\hline V. polyanthes Less. & assa-peixe & $\begin{array}{c}\text { Costa } \\
65\end{array}$ & Fo & Xa, In & $\begin{array}{l}\text { Antiinflamatório, gripe, } \\
\text { tosse }\end{array}$ \\
\hline \multicolumn{6}{|l|}{ Bixaceae } \\
\hline Bixa orellana L. & urucum & $\begin{array}{l}\text { Costa } \\
49\end{array}$ & Se, Fo & $X a, \ln$ & Bronquite \\
\hline \multicolumn{6}{|l|}{ Boraginaceae } \\
\hline Symphytum officinale L. & confrei & $\begin{array}{l}\text { Costa } \\
238\end{array}$ & Fo & $\mathrm{Ct}, \mathrm{In}$ & Queimadura, infecção \\
\hline \multicolumn{6}{|l|}{ Brassicaceae } \\
\hline Brassica rapa L. & couve & $\begin{array}{c}\text { Costa } \\
39\end{array}$ & Fo & $\mathrm{Su}$ & Úlcera, dor de estômago \\
\hline \multicolumn{6}{|l|}{ Celastraceae } \\
\hline Maytenus aquifolium Mart. & $\begin{array}{l}\text { espinheira- } \\
\text { santa }\end{array}$ & $\begin{array}{c}\text { Costa } \\
146\end{array}$ & Fo & In, De & $\begin{array}{l}\text { Mau olhado, dor de } \\
\text { estômago }\end{array}$ \\
\hline \multicolumn{6}{|l|}{ Convolvulaceae } \\
\hline Ipomoea batatas (L.) Lam. & Batata-doce & $\begin{array}{c}\text { Costa } \\
123 \\
\end{array}$ & Fo & De, In & $\begin{array}{l}\text { Antiinflamatório, dor de } \\
\text { dente }\end{array}$ \\
\hline \multicolumn{6}{|l|}{ Costaceae } \\
\hline Costus spiralis (Jacq.) Roscoe & $\begin{array}{l}\text { Caninha-do- } \\
\text { brejo }\end{array}$ & $\begin{array}{l}\text { Costa } \\
38\end{array}$ & Fo & In, BA & $\begin{array}{l}\text { Infecção urinária, no ovário } \\
\text { e no útero, irritação nos } \\
\text { ol hos }\end{array}$ \\
\hline \multicolumn{6}{|l|}{ Crassulaceae } \\
\hline Kalanchoe pin nata (Lam.) Pers. & bálsamo & $\begin{array}{c}\text { Costa } \\
113\end{array}$ & Fo & $\mathrm{Na}$ & Para o fígado \\
\hline $\begin{array}{l}\text { Sedum dendroideum Moc. \& } \\
\text { Sessé ex DC. }\end{array}$ & bálsamo & $\begin{array}{c}\text { Costa } \\
51\end{array}$ & Fo & $\begin{array}{l}\text { In, } \mathrm{Na} \\
\mathrm{Ma}, \mathrm{Ct}\end{array}$ & $\begin{array}{l}\text { Cicatrizante, dor de } \\
\text { estômago Gastrite, gases, } \\
\text { in flamação }\end{array}$ \\
\hline \multicolumn{6}{|l|}{ Cucurbitaceae } \\
\hline Sechium edule (Jacq.) Sw. & chuchu & $\begin{array}{c}\text { Costa } \\
182\end{array}$ & Fo, Fr & $\mathrm{Na}, \mathrm{In}$ & Taquicardia, pressão alta \\
\hline \multicolumn{6}{|l|}{ Equisetaceae } \\
\hline Equisetum hyemale L. & bambuzinho & $\begin{array}{c}\text { Costa } \\
157 \\
\end{array}$ & Fo & In & Cálculo renal, para o rim \\
\hline \multicolumn{6}{|l|}{ Euph orbiaceae } \\
\hline Croton zehntneri Pax. \& K. Hoffm. & $\begin{array}{l}\text { canela-de- } \\
\text { ema }\end{array}$ & $\begin{array}{c}\text { Costa } \\
162\end{array}$ & Fo & Al & Dor muscular, câimbra \\
\hline Jatropha curcas L. & $\begin{array}{l}\text { pinhãozi nho- } \\
\text { da-india }\end{array}$ & $\begin{array}{l}\text { Costa } \\
215\end{array}$ & Se & $\mathrm{De}$ & Afinar o sang ue \\
\hline
\end{tabular}


TABELA 1. Espécies utilizadas como medicinais pela comunidade do bairro dos Tenentes, Extrema, MG.

... continuação

\begin{tabular}{|c|c|c|c|c|c|}
\hline $\begin{array}{l}\text { Família } \\
\text { Espécie }\end{array}$ & $\begin{array}{l}\text { Nome } \\
\text { comum }\end{array}$ & $\begin{array}{l}\text { No de } \\
\text { coleta }\end{array}$ & $\begin{array}{c}\text { Parte } \\
\text { utilizada }\end{array}$ & $\begin{array}{c}\text { Formas } \\
\text { de } \\
\text { preparo }\end{array}$ & Indicações \\
\hline \multicolumn{6}{|l|}{ Euphorbiaceae } \\
\hline J. gossypiffolia L. & pinhão-roxo & $\begin{array}{c}\text { Costa } \\
46 \\
\end{array}$ & Fo & $\mathrm{Ba}$ & Alergia de pele \\
\hline \multicolumn{6}{|l|}{ Fabaceae } \\
\hline Acacia farnesiana (L) Willd. & colônia & $\begin{array}{l}\text { Costa } \\
72\end{array}$ & Se, Fo & De, In & Paralisia facial \\
\hline $\begin{array}{l}\text { Bauhinia cheilantha (Bong.) } \\
\text { Steud. }\end{array}$ & $\begin{array}{l}\text { pata-de-vaca } \\
\text { branca }\end{array}$ & $\begin{array}{l}\text { Costa } \\
156\end{array}$ & Fo & In & Diabetes \\
\hline B. forficata Link & pata-de-vaca & $\begin{array}{c}\text { Costa } \\
37\end{array}$ & Fo & In & Diabetes \\
\hline \multicolumn{6}{|l|}{ Lam iaceae } \\
\hline Hyptis suaveolens (L.) Poit. & $\begin{array}{l}\text { cidrão, } \\
\text { cidreira-de- } \\
\text { folha }\end{array}$ & $\begin{array}{l}\text { Costa } \\
202\end{array}$ & Fo & In & Calmante, gripe \\
\hline Leonotis nepetifolia (L.) R. Br. & rubi & $\begin{array}{l}\text { Costa } \\
217\end{array}$ & Fo & Ma, In & Ferida, diabetes \\
\hline Leonurus sibiricus L. & rubi & $\begin{array}{c}\text { Costa } \\
87\end{array}$ & Fo & $\mathrm{Ma}$ & Cicatrizante \\
\hline Melissa officinalis L. & cidreir a & $\begin{array}{l}\text { Costa } \\
79\end{array}$ & Fo & In & Pressão alta \\
\hline Mentha spp. & $\begin{array}{l}\text { hortelã, } \\
\text { menta, poejo }\end{array}$ & $\begin{array}{c}\text { Costa } \\
199 / 216\end{array}$ & Fo, $\mathrm{Na}$ & $\ln$ & $\begin{array}{l}\text { Dor de barriga gripe, tosse, } \\
\text { febre, verme, calmante, } \\
\text { enjôo (cria nça), dor de } \\
\text { estômago, úlcera }\end{array}$ \\
\hline M. arvensis $\mathrm{L}$. & Hortelã, poejo & $\begin{array}{c}\text { Costa } \\
138\end{array}$ & Fo & Xá In & $\begin{array}{l}\text { Resfriado, tosse, bronquite } \\
\text { dor de barriga }\end{array}$ \\
\hline Mentha X piperita L. & $\begin{array}{l}\text { hor telã- } \\
\text { pimenta, } \\
\text { hortelã }\end{array}$ & Costa 8 & Fo & $\mathrm{Xa}, \ln$ & Gripe, tosse \\
\hline Mentha X villosa Huds. & hortelã & $\begin{array}{c}\text { Costa } \\
36\end{array}$ & Fo & $\ln$ & Gripe, tosse \\
\hline Ocimum gratissimum $\mathrm{L}$. & favacão & $\begin{array}{c}\text { Costa } \\
52\end{array}$ & Fo & $\ln$ & Gripe \\
\hline O. canum Sims. & manjericão & $\begin{array}{l}\text { Costa } \\
80\end{array}$ & Fo, FI & In, Xa & Calmante, bronquite \\
\hline Plectranthus barbatus Andrews & boldo & $\begin{array}{c}\text { Costa } \\
09\end{array}$ & Fo & $\mathrm{Ma}, \mathrm{Na}$ In & $\begin{array}{l}\text { Má digestão, dor de } \\
\text { estômago, para o fígado, } \\
\text { "ressaca" }\end{array}$ \\
\hline P. ornatus Codd. & $\begin{array}{l}\text { boldo, boldo- } \\
\text { de-chão, } \\
\text { estomal ina }\end{array}$ & $\begin{array}{c}\text { Costa } \\
21\end{array}$ & Fo & $\mathrm{Ma}, \ln$ & $\begin{array}{l}\text { Dor de estômago, para o } \\
\text { fígado }\end{array}$ \\
\hline Rosmarinus officinalis $\mathrm{L}$. & alecrim & $\begin{array}{c}\text { Costa } \\
153\end{array}$ & $\mathrm{Ra}$ & $\mathrm{Ba}, \mathrm{In}$ & $\begin{array}{l}\text { Falta de ar, calmante, } \\
\text { angústia, mal estar, mau } \\
\text { ol hado }\end{array}$ \\
\hline \multicolumn{6}{|l|}{ Lauraceae } \\
\hline Ocotea odorifera (Vell.) Rohwer & canela & $\begin{array}{c}\text { Costa } \\
64\end{array}$ & Fo & $\mathrm{Al}$ & Dor externa \\
\hline Persea americana Mill. & abacate & $\begin{array}{c}\text { Costa } \\
44\end{array}$ & Fo & In & Infecção urinária, para o rim \\
\hline
\end{tabular}


TABELA 1. Espécies utilizadas como medicinais pela comunidade do bairro dos Tenentes, Extrema, MG.

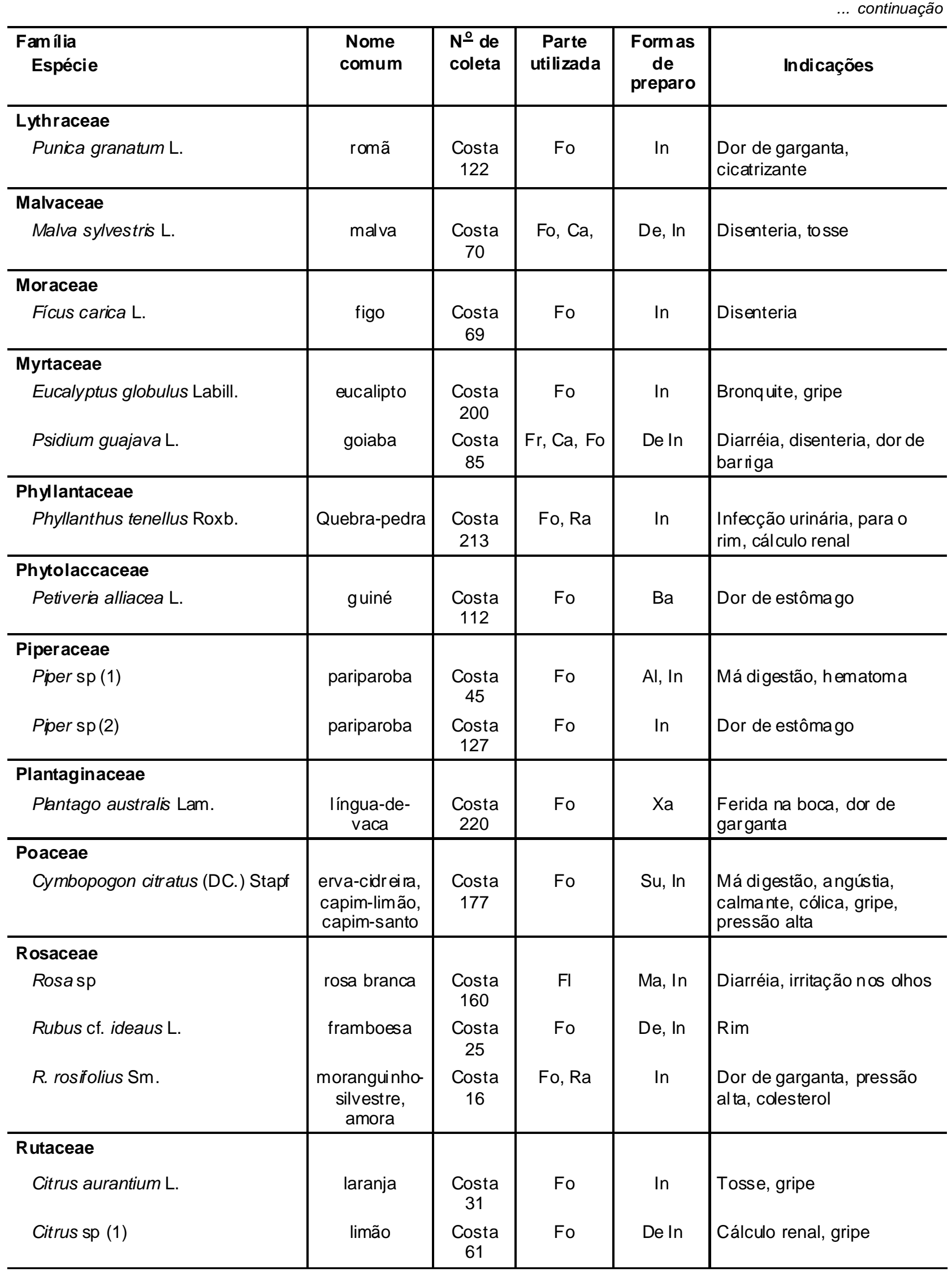


TABELA 1. Espécies utilizadas como medicinais pela comunidade do bairro dos Tenentes, Extrema, MG.

\begin{tabular}{|c|c|c|c|c|c|}
\hline $\begin{array}{l}\text { Fam ília } \\
\text { Espécie }\end{array}$ & $\begin{array}{l}\text { Nome } \\
\text { comum }\end{array}$ & $\begin{array}{l}\mathrm{N} \text { ㅇ de } \\
\text { coleta }\end{array}$ & $\begin{array}{c}\text { Parte } \\
\text { utilizada }\end{array}$ & $\begin{array}{c}\text { Formas } \\
\text { de } \\
\text { preparo }\end{array}$ & Indicações \\
\hline \multicolumn{6}{|l|}{ Rutaceae } \\
\hline Citrus sp (2) & limão & $\begin{array}{c}\text { Costa } \\
124\end{array}$ & $\mathrm{Fr}$ & $\mathrm{Xa}$ & Tosse \\
\hline Ruta graveolens L. & arruda & $\begin{array}{c}\text { Costa } \\
81\end{array}$ & $\mathrm{Ra}$ & $\begin{array}{l}\text { In, Ba } \\
\mathrm{Ma}, \mathrm{In}\end{array}$ & $\begin{array}{l}\text { Dor no corpo, mau olhado, } \\
\text { conjuntivite, cólica } \\
\text { menstrual, purifica o sangue }\end{array}$ \\
\hline \multicolumn{6}{|l|}{ Urticaceae } \\
\hline Cecropia pachystachya Trécul & embaúba & $\begin{array}{c}\text { Costa } \\
19\end{array}$ & Fo & In & Hipertensão \\
\hline \multicolumn{6}{|l|}{ Verbenaceae } \\
\hline Lantana camara L. & cambará & $\begin{array}{c}\text { Costa } \\
144\end{array}$ & Fo & In & Resfriado \\
\hline Lippia alba (Mill.) N.E. Br. & cidreir a & $\begin{array}{c}\text { Costa } \\
125\end{array}$ & Fo & In & Calmante \\
\hline $\begin{array}{l}\text { Stachytarpheta cayennensis } \\
\text { (Rich.) Vahl }\end{array}$ & gerbão & $\begin{array}{c}\text { Costa } \\
32\end{array}$ & $\mathrm{FI}, \mathrm{Fo}$ & $\ln$ & Fígado \\
\hline \multicolumn{6}{|l|}{ Vitaceae } \\
\hline $\begin{array}{l}\text { Cissus verticillata (L.) Nicolson \& } \\
\text { C.E. Jarvis }\end{array}$ & insulina & $\begin{array}{l}\text { Costa } \\
50\end{array}$ & Fo & In & Diabetes \\
\hline \multicolumn{6}{|l|}{ Zingiberaceae } \\
\hline $\begin{array}{l}\text { Alpinia zerumbet (Pers.) B.L. Burtt. } \\
\text { \& R.M. Sm. }\end{array}$ & pacová & $\begin{array}{c}\text { Costa } \\
167\end{array}$ & $\mathrm{Se}$ & $\mathrm{De}$ & Vermes \\
\hline
\end{tabular}

Partes utilizadas: raízes $(\mathrm{Rz})$, caules $(\mathrm{Ca})$,folhas $(\mathrm{Fo})$, flores $(\mathrm{Fl})$, frutos $(\mathrm{Fr})$, sementes $(\mathrm{Se})$. Formas de preparo: infusão (In), decocção $(\mathrm{De})$, xarope $(\mathrm{Xa})$, in natura $(\mathrm{Na})$, alcoolatura $(\mathrm{Al})$, banho $(\mathrm{Ba})$, cataplasma $(\mathrm{Ct})$, maceração $(\mathrm{Ma})$, suco $(\mathrm{Su})$.

Quanto aos problemas relacionados ao sistema nervoso, algumas espécies foram citadas como "calmantes". Entre essas, Os extratos de Chamomilla recutita (L.) Rauschet, Cymbopogon citratuse Lippia alba (Mill.) N.E. Br. tiveram a atividade sedativa confirmada em testes com cobaias (Della Loggia et al., 1982; Hennebelle et al., 2008; Blanco et al., 2009). Estudos mostram atividade sedativa em extratos de Ocimum gratissimum (Freire et al., 2006), o que também poderia ocorrer com $O$. canum, indicada também como calmante pelos entrevistados.

Hipertensão foi a única doença relacionada ao aparelho circulatório, para qual algumas espécies foram indicadas, porém apenas Sechium edule (Jacq.) Sw. teve atividade hipotensiva confirmada em testes de laboratório (Gordon et al., 2000). Entre as plantas citadas para pressão alta estão também Melissa officinalis L. e Cimbopogon citratus, cujos extratos aquosos geraram redução da frequência cardíaca em cobaias (Gazola et al., 2004), o que também poderia contribuir para o controle da pressão arterial, corroborando as indicações feitas pelos entrevistados.

Entre as plantas utilizadas para o tratamento do aparelho reprodutor, Costus spiralis foi apontada para o tratamento de infecção no ovário e útero, e Ruta graveolens para cólicas menstruais. A atividade antibacteriana de $C$. spiralis foi observada por Perez et al. (2008) e $R$. graveolens teve a atividade antiespasmódica (Minker et al., 1979), potenciais anticonceptivo (Gandhi et al., 1991, Gutiérrez-Pajares et al., 2003) e abortivo (Freitas et al., 2005) avaliados em testes com cobaias, atividades relacionadas a ação emenagoga, corroborando as indicações propostas pelos entrevistados.

Punica granatum e Schinus terebinthifolia Raddi foram citadas como cicatrizantes. Estudos com cobaias confirmam sua ação cicatrizante (Murthy et al., 2004; Branco-Neto et al., 2006), enquanto Sedum dendroideume Leonurus sibiricus tiveram a atividade antiinflamatória confirmada por De Melo et al. (2005) e Islam et al. (2005), respectivamente. Symphytum officinale e Aloe vera (L.) Burm. f. foram indicadas pelos entrevistados para tratamento de queimaduras. No extrato de S. officinale, constituintes como a alantoina geram proliferação celular epitelial, contribuindo para cicatrização de áreas lesadas (Barbakadze et al., 2009), e em $A$. vera, a mucilagem (aloeferon) tem ação cicatrizante em queimaduras (Maenthaisongab et al., 2007), 
corroborando o uso dessas espécies pela comunidade, para o tratamento de lesões na pele e queimaduras.

Porophyllum ruderale (Jacq.) Cass., Ipomoea batatas L. (Lam.), Sedum dendroideum e Vernonia polyanthesforam indicadas como antiinflamatórios. Entre elas, atividade antiinflamatória in vitrofoi observada com o óleo essencial de P. ruderale (Souza et al., 2003) e com extratos de $V$. polyanthes (Barbastefano et al., 2007), S. dendroideum (De Melo et al., 2005), corroborando o uso dessas espécies pelos entrevistados. Porém, segundo Perez \& Hakumata (1999), o extrato produzido com folhas de I. batatas não apresentou atividade antiinflamatória segundo o modelo experimental utilizado.

Para dores externas e musculares foram apontadas Ocotea odorifera (Vell.) Rohwer, Ruta graveolens, Artemisia annua L., A. canphorata Vill. e Croton zehntneri Pax. \& K. Hoffm. Todas as espécies apresentam na composição óleos essenciais, os quais poderiam contribuir para uma analgesia local, porém esta atividade só foi observada em cobaias com extratos de C. zehntneri (Oliveira et al., 2001).

As partes vegetais mais utilizadas no preparo das plantas medicinais foram as folhas e ramos (88\%). As demais partes vegetais tiveram uma utilização igual ou inferior a $5 \%$, o que é também observado em outros levantamentos etnobotânicos (Pinto et al., 2006; CunhaLima et al., 2008; Jesus et al., 2009). Segundo Martin (1995), a preferência pelo uso das folhas apresenta um caráter de conservação do recurso vegetal, pois quando feito de maneira adequada, preserva a espécie utilizada. As formas de preparo mais utilizadas pela comunidade foram infusão (67\%), decocção (9\%) e xarope (7\%), resultados semelhantes também foram observados em outros levantamentos (Medeiros et al., 2004; Pereira et al., 2004; Vendruscolo \& Mentz, 2006).

Através deste estudo observou-se ainda que 0 conhecimento medicinal das plantas, na comunidade, vem sendo adquirido e transmitido de geração a geração por familiares (58\%), amigos e vizinhos (38\%) e através da literatura e meios de comunicação (4\%). Dos 50 entrevistados, $84 \%$ citaram as plantas medicinais no tratamento de várias enfermidades, $12 \%$ utilizam as plantas para algumas enfermidades específicas e 4\% dos entrevistados declararam utilizar apenas plantas medicinais para suas enfermidades, não recorrendo a nenhuma outra forma de tratamento. A utilização das plantas medicinais pela maioria dos entrevistados foi relacionada à precariedade do sistema de saúde da cidade, ou à distância para buscar atendimento médico. $\mathrm{Na}$ literatura consultada foram encontrados trabalhos que corroboram as indicações de 52 espécies, o que corresponde aproximadamente a $75 \%$ das espécies identificadas.

Desta forma, este trabalho evidenciou que parte expressiva das indicações propostas pelos entrevistados sobre as espécies utilizadas já foi avaliada experimentalmente, ainda que de forma preliminar, podendo ser fonte promissora para descoberta de novos princípios ativos.

\section{REFERÊNCIA}

ALBUQUERQUE, U.P.; HANAZAKI, N. As pesquisas etnodirigidas na descoberta de novos fármacos de interesse médico e farmacêutico: fragilidades e perspectivas. Revista Brasileira de Farmacognosia, v.16, supl., p.678-89, 2006.

ALBUQUERQUE, U.P.A.; LUCENA, R.F.P. Métodos e técnicas para coleta de dados. In: ALBUQUERQUE, U.P.A.; LUCENA, R.F.P. (Orgs.). Métodos e técnicas na pesquisa etnobotânica. Recife: Livro Rápido/NUPEEA, 2004. p.37-62.

AMOROZO M.C.M. A abordagem etnobotânica na pesquisa de plantas medicinais. In: DI STASI, L.C. (Org.). Plantas medicinais arte e ciência: um guia de estudo interdisciplinar. São Paulo: Editora da Universidade Estadual Paulista, 1996. p.47-68.

AREF, H.L. et al. In vitro antimicrobial activity of four Ficus carica latex fractions against resistant human pathogens.

Pakistan Journal of Pharmaceutical Sciences, v.23, n.1, p.53-8, 2010.

ARNOUS, A.H.; SANTOS, A.S.; BEINNER, R.P.C. Plantas medicinais de uso caseiro - conhecimento popular e interesse por cultivo comunitário. Revista Espaço para a Saúde, v.6, n.2, p.1-6, 2005.

BARBAKADZE, V. et al. Allantoin and pyrrolizidine alkaloids-free wound healing compositions from Symphytum asperum. Bulletin of the Georgian National Academy of Sciences, v.3, n.1, p.159-64, 2009.

BARBASTEFANO, V. et al. Vernonia polyanthes as a new source of antiulcer drugs. Fitoterapia, v.78, n.7-8, p.54551, 2007.

BARBOSA, W.L.R. et al. Flavonóides de Cissus verticillata e a atividade hipoglicemiante do chá de suas folhas. Revista Brasileira de Farmacognosia, v.12, supl.1, p.135, 2002.

BLANCO, M.M. et al. Neurobehavioral effect of essential oil of Cymbopogon citratus in mice. Phytomedicine, v.16, n.23, p.265-70, 2009.

BRANCO-NETO, M.L.C. et al. Avaliação do extrato hidroalcoólico de aroeira (Schinus terebinthifolius Raddi) no processo de cicatrização de feridas em pele de ratos.

Acta Cirúrgica Brasileira, v.21, supl.2, p.17-22, 2006.

CÂMARA, C.C. et al. Antispasmodic effect of the essential oil of Plectranthus barbatus and some major constituents on the guinea-pig ileum. Planta Medica, v.69, n.12, p.1080-5, 2003.

CAMPOS, A.H.; SCHOR, N. Phyllanthus niruri inhibits calcium oxalate endocytosis by renal tubular cells: its role in urolithiasis. Nephron Journal, v.81, n.4, p.393-7, 1999. CASTILLO-JUÁREZ, I. et al. Anti-Helicobacter pylori activity of plants used in Mexican traditional medicine for gastrointestinal disorders. Journal of Ethnopharmacology, v.122, n.2, p.402-5, 2009.

CHENG, C.; WANG, Z. Bacteriostatic activity of anthocyanin of Malva sylvestris. Journal of Forestry Research, v.17, n.1, p.83-5, 2006.

CUNHA-LIMA, S.T. et al. Levantamento da flora medicinal

Rev. Bras. Pl. Med., Botucatu, v.13, n.3, p.282-292, 2011. 
usada no tratamento de doenças metabólicas em Salvador, BA, Brasil. Revista Brasileira de Plantas Medicinais, v.10, n.4, p.83-9, 2008.

DELLA LOGGIA, R. et al. Depressive effects of Chamomilla recutita (L.) Rausch tubular flowers on central nervous system in mice. Pharmacological Research Communications, v.14, n.2, p.153-62, 1982. DE MELO, G.O. et al. Phytochemical and pharmacological study of Sedum dendroideum leaf juice. Journal of Ethnopharmacology, v.102, n.2, p.217-20, 2005.

EMERENCIANO, V.P. et al. Um novo método para agrupar parâmetros quimiotaxonômicos. Química Nova, v.21, n.2, p.125-9, 1998.

FIDALGO, O.; BONONI, V.L.R. Técnicas de coleta e herborização de material botânico. 2.ed. São Paulo: Instituto de Botânica, 1989. 62p.

FONSECA-KRUEL, V.S.; PEIXOTO, A.L. Etnobotânica na reserva extrativista marinha de Arraial do Cabo, RJ, Brasil. Acta Botânica Brasilica, v.18, n.1, p.177-90, 2004. FRANCO, E.A.P.; BARROS, R.F.M. Uso e diversidade de plantas medicinais no Quilombo Olho D'água dos Pires, Esperantina, Piauí. Revista Brasileira de Plantas Medicinais, v.8, n.3, p.78-88, 2006.

FREIRE, C.M.M.; MARQUES, M.O.M.; COSTA, M. Effects of seasonal variation on the central nervous system activity of Ocimum gratissimum L. essential oil. Journal of Ethnopharmacology, v.105, n.1-2, p.161-6, 2006.

FREITAS, A.M.; SCHOR, N.; BOIM, M.A. The effect of Phyllanthus niruri on urinary inhibitors of calcium oxalate crystallization and other factors associated with renal stone formation. British Journal of Urology, v.89, n.8, p.829-34, 2002.

FREITAS, T.; AUGUSTO, P.; MONTANARI, T. Effect of Ruta graveolens L. on pregnant mice. Contraception, v.71, n.1, p.74-7, 2005.

FRUTUOSO, V.S. et al. Analgesic and anti-ulcerogenic effects of a polar extract from leaves of Vernonia condensata. Planta Medica, v.60, n.1, p.21-5, 1994.

GANDHI, M. et al. Post-coital antifertility action of Ruta graveolens in female rats and hamsters. Journal of Ethnopharmacology, v.34, n.1, p.49-59, 1991.

GAZOLA, R. et al. Lippia alba, Melissa officinalis and Cymbopogon citratus: effects of the aqueous extracts on the isolated hearts of rats. Pharmacological Research, v.50, n.5, p.477-80, 2004.

GENÉ, R.M. et al. Anti-inflammatory and analgesic activity of Baccharis trimera: identification of its active constituents. Planta Medica, v.62, n.3, p.232-5, 1996.

GONZALEZ, F. et al. Antiulcerogenic and analgesic effects of Maytenus aquifolium, Sorocea bomplandii and Zolernia ilicifolia. Journal of Ethnopharmacology, v.77, n.1, p.41-7, 2001.

GORDON, E.A.; GUPPY, L.J.; NELSON, M. The antihypertensive effects of the Jamaican Cho-Cho (Sechium edule). West Indian Medical Journal, v.49, n.1, p.27-31, 2000.

GUTIÉRREZ-PAJARES', J.L.; ZÚÑIGA, L.; PINO, J. Ruta graveolens aqueous extract retards mouse preimplantation embryo development. Reproductive Toxicology, v.17, n.6, p.667-72, 2003.

HANAZAKI, N. et al. Diversity of plant uses in two caiçara communities from the atlantic forest coast, Brazil. Biodiversity and Conservation, v.9, n.6, p.597-615, 2000.
HENNEBELLE, T. et al. Antioxidant and neurosedative properties of polyphenols and iridoids from Lippia alba. Phytotherapy Research, v. 22, n.2, p.256-8, 2008.

IMAI, H. et al. Inhibition by the essential oils of peppermint and spearmint on the growth of pathogenic bacteria. Microbios, v.106, n.1, p.31-9, 2001.

ISLAM, M.A. et al. Analgesic and anti-inflammatory activity of Leonurus sibiricus. Fitoterapia, v.76, n.3-4, p.359-62, 2005.

IVANOVA, A. et al. Antimicrobial and cytotoxic activity of Ruta graveolens. Fitoterapia, v.76, n.3-4, p.344-7, 2005. JEONG, M. et al. Antimicrobial activity of methanol extract from Ficus carica leaves against oral bacteria. Journal of Bacteriology and Virology, v.39, n.2, p.97-102, 2009. JESUS, N.Z.T. et al. Levantamento etnobotânico de plantas popularmente utilizadas como antiúlceras e antiinflamatórias pela comunidade de Pirizal, Nossa Senhora do Livramento-MT, Brasil. Revista Brasileira de Farmacognosia, v.19, n.1, p.130-9, 2009.

KHALIL, N.M.; PEPATO, M.T.; BRUNETTI, I.L. Free radical scavenging profile and myeloperoxidase inhibition of extracts from antidiabetic plants: Bauhinia forficata and Cissus sicyoides. Biological Research, v.41, n.2, p.165-71, 2008. LAPENNA, E.A. et al. Actividad bactericida y fungicida de algunas plantas utilizadas en la medicina tradicional venezolana. Revista del Instituto Nacional de Higiene Rafael Rangel, v.34, n.1, p.6-9, 2003.

LEMMENS-GRUBER, R. et al. Investigation of the spasmolytic activity of the flavonoid fraction of Achillea millefolium on isolated guinea-pig ilea. Arzneimittelforschung Journal, v.56, n.8, p.582-8, 2006.

LOPES-MARTINS, R.A.B. et al. The anti-inflammatory and analgesic effects of a crude extract of Petiveria alliacea $\mathrm{L}$. (Phytolaccaceae). Phytomedicine, v.9, n.3, p.245-8, 2002. LORENZETTI, B.B. et al. Myrcene mimics the peripheral analgesic activity of lemongrass tea. Journal of Ethnopharmacology, v.34, n.1, p.43-8, 1991.

LOZOYA, X. et al. Quercetin glycosides in Psidium guajava $\mathrm{L}$. leaves and determination of a spasmolytic principle. Archives of Medical Research, v.25, n.1, p.11-5, 1994. MACIEL, M.A.M. et al. Plantas medicinais: a necessidade de estudos multidisciplinares. Quimica Nova, v.25, n.3, p.429-38, 2002.

MACEDO, M.; CARVALHO, J.M.K.; NOGUEIRA, F.L. Plantas medicinais e ornamentais da área de aproveitamento múltiplo de Manso, Chapada dos Guimarães, Mato Grosso. Cuiabá: Ed. da UFMT, 2002. $188 p$.

MAENTHAISONGAB, R. et al. The efficacy of Aloe vera used for burn wound healing: a systematic review. Journal of the International Society for Burns Injuries, v.33, n.6, p.713-8, 2007.

MANS, D. et al. Assessment of eight popularly used plantderived preparations for their spasmolytic potential using the isolated guinea pig ileum. Pharmaceutical Biology, v.42, n.6, p.422-9, 2004.

MARGORTE SILVA, M.J.; CAPAZ, F.R.; VALE, M.R. Effects of the water soluble fraction from leaves of Ageratum conyzoides on smooth muscle. Phytotherapy Research, v.14, n.2, p.130-2, 2000.

MARTIN, G.J. Ethnobotany: a 'people and plants' conservation manual. London: Chapman \& Hall, 1995. $268 p$. 
MAURO, C. et al. Estudo botânico, fitoquímico e avaliação da atividade antimicrobiana de Rubus rosaefolius Sm. Rosaceae. Revista Brasileira de Farmacognosia, v.12, supl., p.23-5, 2002.

MEDEIROS, M.F.T.; FONSECA, V.S.; ANDREATA, R.H.P. Plantas medicinais e seus usos pelos sitiantes da Reserva Rio das Pedras, Mangaratiba, RJ, Brasil. Acta Botanica Brasílica, v.18, n.2, p.391-9, 2004.

MELLO, F.B.; MELLO, J.R.B. Efeitos antitussígenos e expectorantes de dois fitoterápicos comercializados no mercado brasileiro. Acta Scientiae Veterinariae, v.33, n.2, p.161-7, 2005.

MENEZES, F.S. et al. Hypoglycemic activity of two Brazilian Bauhinia species: Bauhinia forficata L. and Bauhinia monandra Kurz. Revista Brasileira de Farmacognosia, v.17, n.1, p.8-13, 2007.

MESIA-VELA, S. et al. Pharmacological study of Stachytarpheta cayennensis (Rich.) Vahl in rodents. Phytomedicine, v.11, n.7-8, p.616-24, 2004.

MINKER, E. et al. Pharmacological study of the antispasmodic principles isolated from Ruta graveolens L. (rue). Planta Medica, v.36, n.3, p.255-6, 1979.

MURTHY, K.N. et al. Study on wound healing activity of $P$ unica granatum peel. Journal of Medicinal Food, v.7, n.2, p.2569, 2004.

NARANJO, J.P. et al. Actividad antiparasitaria de una decocción de Mentha piperita Linn. Revista Cubana de Medicina, v.35, n.1, p.1-7, 2006.

NAZ, S. et al. Antibacterial activity directed isolation of compounds from Punica granatum. Journal of Food Science, v.72, n.4, p.341-5, 2007.

OLIVEIRA, A.C. et al. Antinociceptive effects of the essential oil of Croton zehntneriin mice. Brazilian Journal of Medical and Biological Research, v.34, n.11, p.1471-4, 2001.

OLIVEIRA, D.F. et al. Antibacterial activity of plant extracts from Brazilian southeast region. Fitoterapia, v.78, n.2, p.1425, 2007a.

OLIVEIRA, D.G. et al. Antimycobacterial activity of some Brazilian indigenous medicinal drinks. Revista de Ciências Farmacêuticas Básica e Aplicada, v.28, n.2, p.165-9, 2007b. OLIVEIRA, F.C. et al. Avanços nas pesquisas etnobotânicas no Brasil. Acta Botanica Brasileira, v.23, n.2, p.590-605, 2009.

PEPATO, M.T. et al. Anti-diabetic activity of Bauhinia forficata decoction in streptozotocin-diabetic rats. Journal of Ethnopharmacology, v.81, n.2, p.191-7, 2002.

PEREIRA, R.C.; OLIVEIRA, M.T.R.; LEMOS, G.C.S. Plantas utilizadas como medicinais no município de Campos de Goytacazes - RJ. Revista Brasileira de Farmacognosia, v.14, supl., p.37-40, 2004.

PEREZ, A.C.; HAKUMATA, L.Y. Efeito antiinflamatório da folha de batata-doce (Ipomoea batatas). Revista de Odontologia UNESP, v.28, n.1, p.161-5, 1999.

PÉREZ, C. et al. Antibacterial effect of Costus spiralis leaves extract on pathogenic strains of Vibrio cholerae. Revista Cenic: Ciencias Biológicas, v.39, n.1, p.70-2, 2008.

PHILLIPS, O.; GENTRY, A.M. The useful plants of Tambopata, Peru. I. Statistical hypothesis with a new quantitative technique. Economic Botany, v.47, n.1, p.15-32, 1993.

PINTO, E.P.P.; AMOROZO, M.C.M.; FURLAN, A. Conhecimento popular sobre plantas medicinais em comunidades rurais de mata atlântica - Itacaré, BA, Brasil.
Acta Botânica Brasílica, v.20, n.4, p.751-62, 2006. RIBEIRO, D. Observando as sub-bacias hidrográficas de extrema-semana da água. Extrema: Grilo \& Grilo, 2004. $68 p$.

RODRIGUES E.; CARLINI, E.L.A. Levantamento etnofarmacológico realizado entre um grupo de quilombolas do Brasil. Arquivos Brasileiros de Fitomedicina Científica, v.1,n.1, p.80-7, 2003.

RODRIGUES, V.E.G.; CARVALHO, D.A. Levantamento etnobotânico de plantas medicinais no domínio do cerrado na região do Alto Rio Grande-Minas Gerais. Ciência Agrotécnica, v.25, n.1, p.102-23, 2001.

SALGADO, H.R.N. et al. Evaluation of antidiarrhoeal effects of Psidium guajava L. (Myrtaceae) aqueous leaf extract in mice. Revista de Ciências Farmacêuticas Básica e Aplicada, v.27, n.1, p.89-92, 2006.

SANTOS, E.B. et al. Estudo etnobotânico de plantas medicinais para problemas bucais no município de João Pessoa, Brasil. Revista Brasileira de Farmacognosia, v.19, n.1, p.321-4, 2009.

SIMÕES, C.M.O. Plantas da medicina popular no Rio Grande do Sul. 5.ed. Porto Alegre: Ed. da UFRGS, 1998. $173 p$.

SIMÕES, C.M.O.; SPITZER, V. Óleos voláteis. In: SIMÕES, C.M.O. et al. (Orgs.). Farmacognosia: da planta ao medicamento. 2.ed. Porto Alegre/Florianópolis: Ed. UFRGS/ UFSC, 2000. p.387-416.

SOUSA, M.P. et al. Constituintes químicos de plantas medicinais brasileiras. Fortaleza: Ed. da UFC, 1991. 416p. SOUZA, M.C. et al. Evaluation of anti-inflammatory activity of essential oils from two Asteraceae species. Pharmazie, v.58, n.8, p.582-6, 2003.

STEVENS, P.F. APG III. Angiosperm Phylogeny Website. Version 9, June 2008. Disponível em: <http://www. mobot.org/MOBOT/research/APweb>. Acesso em: 10 set. 2010.

TONA, L. et al. Antiamoebic and spasmolytic activities of extracts from some antidiarrhoeal traditional preparations used in Kinshasa, Congo. Phytomedicine, v.7, n.1, p.31-8, 2000.

VENDRUSCOLO, G.S.; MENTZ, L.A. Levantamento etnobotânico das plantas utilizadas como medicinais por moradores do bairro Ponta Grossa, Porto Alegre, Rio Grande do Sul, Brasil. Iheringia: Série Botânica, v.61, n.12, p.83-103, 2006.

VIDAL, F. et al. Giardia lamblia: The effects of extracts and fractions from Mentha $x$ piperita Lin. (Lamiaceae) on trophozoites. Experimental Parasitology, v.115, n.1, p.2531, 2007.

VIEL, T.A. et al. Evaluation of the antiurolithiatic activity of the extract of Costus spiralis Roscoe in rats. Journal of Ethnopharmacology, v.66, n.2, p.193-9, 1999.

WHO. Bulletin of the world health organization. Regulatory situation of herbal medicines. A worldwide review. Genebra: WHO, 1998. 49p.

WHO. International Statistical Classification of Diseases and Related Health Problems 10th Revision. Version for 2007. Disponível em: <http://apps.who.int/lassifications/ apps/icd/icd10online>. Acesso em: 14 set. 2010.

YATSUDA, R. et al. Effects of Mikania genus plants on growth and cell adherence of mutans Streptococcus. Journal of Ethnopharmacology, v.97, n.2, p.183-9, 2005. 